\title{
Effect of Puerarin on EBI after SAH
}

\author{
Xiangwu ZENG ${ }^{1}$, Xiuzhen $\mathrm{XU}^{2 *}\left(\mathbb{0}\right.$, Jianlong $\mathrm{KONG}^{1}$, Congxue RONG ${ }^{1}$, Jianhu $\mathrm{SHE}^{1}$, \\ Wanliang $\mathrm{GUO}^{1}$, Lijuan $\mathrm{SHI}^{1}$, Dianfan $\mathrm{ZHAO}^{1}$
}

\begin{abstract}
Objective: The neuroinflammatory reaction activated after the activation of microglia plays a critical role in the pathological mechanism of early brain injury after subarachnoid hemorrhage. The inhibitory effect of Puerarin on inflammatory response plays a protective effect against various diseases. The present study mainly explored whether Puerarin alleviates the inflammation after SAH by inhibiting the Notchl pathway. Methods: Rat SAH model was induced by internal carotid artery puncture. The content of brain edema was evaluated and assessed. Evans blue staining was used to detect the degree of blood-brain barrier damage. FJC staining was used to assess neuronal apoptosis. Western blot method was employed to detect the expressions of Notchl receptor and downstream factors to clarify the protective effect and specific mechanism of Puerarin on brain tissue after SAH. Results: Puerarin can attenuate the brain edema and blood-brain barrier damage caused by early brain injury within $72 \mathrm{~h}$ after SAH, reduce the contents of Ibal and CD68 positive microglia. Intraventricular injection of Notchl receptor blockers and Notchl siRNA can inhibit the activation of Notchl pathway after SAH, mitigate brain tissue edema, blood-brain barrier damage, and neuronal apoptosis, as well as inhibit the activation of microglia and the release of inflammatory factors. Puerarin can inhibit the activation of Notchl receptor and downstream pathways, and simultaneously increase the content of Botch in brain tissue. Conclusion: Puerarin can inhibit the activation of Notchl receptor through Botch factor, thereby inhibiting the neuroinflammatory reaction mediated by Notchl pathway, and finally improve the early neurological dysfunction after SAH.
\end{abstract}

Keywords: Puerarin; EBI; SAH.

Practical Application: Effect of Puerarin on EBI after SAH.

\section{Introduction}

Subarachnoid hemorrhage $(\mathrm{SAH})$ refers to a pathological change caused by blood entering the subarachnoid space. Spontaneous SAH is most commonly caused by bleeding from a ruptured intracranial aneurysm. The mortality rate of first-time bleeding is $40 \%$, while the mortality rate of rebleeding is as high as $40-75 \%$, seriously endangering human life and health, and causing a huge social burden (Minhas et al., 2019; Ballini et al., 2018; Fan et al., 2016).

EBI refers to the pathophysiological process that occurs within $72 \mathrm{~h}$ after SAH. Due to the sharp increase of intracranial pressure and the decrease of cerebral perfusion pressure, blood cells and metabolites enter the subarachnoid space, producing cytotoxic reactions, causing depolarization of the cerebral cortex, oxidative stress, inflammation, apoptosis, autophagy and other cell metabolic response, eventually leading to nerve cell death, blood-brain barrier destruction, brain edema and neurological dysfunction. However, the complex signaling pathways and molecular mechanisms involved in EBI still need further research (Ferri et al., 2016).

Notch receptor is a transmembrane receptor, including four subtypes of Notchl, Notch2, Notch3, and Notch4 in humans. The
Notchl pathway was first discovered in the genetic research of Drosophila. Notchl is a highly conserved intracellular signaling pathway that plays an important role in the process of biological evolution and organ growth and development. Botch is a neuroprotective gene discovered during functional screening of neurons pretreated with hypoxia. Botch has $\gamma$-glutamate transferase activity, which can de-glycine the Notchl receptor, thus avoiding the cleavage of Notchl by S1furin-like invertase and blocking the maturation process of Notchl receptor. The paper investigated whether Botch is involved in the neuroprotective process of Puerarin in SAH (Yang et al., 2017b; Carbonara et al., 2018; Xu et al., 2018).

\section{Methods}

\subsection{Internal carotid artery puncture method to establish SD rat SAH model and grouping}

The cleaned SD male rats were weighed, and anesthetized with $3 \%$ pentobarbital sodium at a dose of $45 \mathrm{mg} / \mathrm{kg}$ for tracheal intubation. The anterior tracheal fascia of the carotid triangle was opened to expose the carotid sheath, the internal common carotid artery, the internal jugular vein, and the vagus nerve.

${ }^{1}$ Department of Brain, The Second People's Hospital of Zhangye City, Zhangye, Gansu, China

${ }^{2}$ Department of Pharmacy, The Second People's Hospital of Zhangye City, Zhangye, Gansu, China

*Corresponding author: xuxiuzhen8193@sina.com 
The ascending pharyngeal artery was found at the beginning of the external carotid artery and at the distal end was the superior thyroid artery, which was then cut with bipolar electrocoagulation. The distal end of the external carotid artery was ligated and straightened. The common carotid artery and neck internal artery were clamped with two vascular clips. Following this, a $1.5 \mathrm{~cm}$ long 5-0 surgical suture were placed between the beginning of the external carotid artery and the ligation point, and the thread pierced through the internal carotid artery bifurcation. The clamp on the common carotid artery was removed. The blood vessel clip was quickly pulled out after about 5 seconds. The stump of the external carotid artery was ligated after electrocoagulation. After operation, the rat was placed on a pre-heated electric blanket and placed in the observation room after waken-up. After $12 \mathrm{~h}$, the rat was transferred to the animal breeding room and fed in a single cage.

The rats were grouped as follows:

A: The Sham group, in which the rats were given sham operation and treated with PBS.

B: The Model group, SAH, the rats were given PBS treatment.

C: The Puerarin low-dose group, SAH, the rats were given $5 \mathrm{mg} / \mathrm{kg}$ Puerarin.

D: The High-dose Puerarin group, $\mathrm{SAH}$, the rats were given $20 \mathrm{mg} / \mathrm{kg}$ Puerarin.

\subsection{Determination of water content in brain tissue}

In the experiment, the wet and dry weight method was used to determine the water content in brain tissue.

\subsection{Detection of blood-brain barrier permeability}

The experiment evaluated the permeability of the bloodbrain barrier through quantitative analysis of Evans blue dye exudation. $72 \mathrm{~h}$ after the establishment of the rat SAH model, weighing and anesthesia were performed. The femoral vein on the left side of the rat was isolated under the microscope, and $2 \%$ EB solution $(4 \mathrm{ml} / \mathrm{kg})$ was injected. Two hours after the injection, $3 \%$ pentobarbital sodium was used to deeply anesthetize the rats again. After perfusion with $250 \mathrm{ml}$ iced saline, the brain was quickly removed through craniotomy. Brain tissue was divided into the left and right hemispheres on a glass plate on the ice box. The left hemisphere was weighed and soaked in $2 \mathrm{ml}$ of $50 \%$ trichloroacetic acid solution, then incubated in a $37^{\circ} \mathrm{C}$ blast drying oven for $72 \mathrm{~h}$. According to the instructions, the EB content in the sample to be tested was calculated.

\subsection{FJC staining}

The brain tissue slices of each group were soaked in FJC working solution for $25 \mathrm{~min}$, then washed with distilled water. Finally, the cleaned brain slices were put into a $50{ }^{\circ} \mathrm{C}$ drying oven to bake for $30 \mathrm{~min}$. The baked slices were soaked for $5 \mathrm{~min}$ in xylene. The slices were taken out and dried in a fume hood and sealed with neutral gum. The slices were observed under a fluorescence microscope according to the instructions.

\subsection{IF staining}

After instilling the materials and paraffin sections, they were repaired with antigen, permeated, closed, incubated with antibodies, and observed under a microscope after DAPI staining.

\subsection{WB method to determine the expression of various proteins}

According to the experimental design, $72 \mathrm{~h}$ after SAH, the rats were deeply anesthetized with $3 \%$ pentobarbital sodium, the heart was perfused with sterile saline, the brain was quickly taken out through craniotomy, and the brain tissue was placed in a cryopreservation tube, and preserved in liquid nitrogen. WB experiment was conducted to detect the contents of Notchl, NICD, RBP-Jk, Hes- 1 and Botch proteins in brain tissue according to the instructions.

\subsection{Statistical analysis}

All data are expressed as mean \pm standard errors. SPSS 19.0 software was used for data analysis. Comparison between two groups was performed using t test and Mann Whitney $U$ test. Comparison between multiple groups was performed using one-way analysis of variance (ANOVA) and Kruskal-Wallis test. $\mathrm{P}<0.05$ was considered statistically significant. GraphPad Prism 5 software was used for drawing.

\section{Result}

\subsection{The effect of Puerarin on cerebral edema}

Determination of water content in brain tissue showed that Puerarin can reduce the degree of edema in brain tissue, see Figure 1.

\subsection{The protective effect of Puerarin on the blood-brain barrier}

After SAH, the Evans blue content in the left cerebral hemisphere was determined. The results showed that, after treatment with different concentrations of drugs, the Evans blue content in the lateral brain tissue after $\mathrm{SAH}$ were reduced, indicating that Puerarin has a certain protective effect on the blood brain barrier, see Figure 2 .

\subsection{The regulatory effect of Puerarin on neuron apoptosis}

The results showed that Puerarin can reduce the number of FJC positive cells after SAH and increase the content of normal neuronal cell marker NeuN, proving that different concentrations of drugs can reduce the degenerative apoptosis of neuronal cells after SAH, see Figure 3.

\subsection{The effect of Puerarin on the activation of microglia after $S A H$}

Immunofluorescence staining results showed that the number of activated microglial cells after SAH was decreased, and the number of worm-like cells increased obviously, while the number 
of resting microglia with more protrusions decreased. CD68, used as a marker for activated microglia, also increased significantly after SAH. CD68 is mainly distributed in the edematous area of brain tissue. However, treatment with different concentrations of drugs reduced CD68-positive activated microglia, and the number of resting microglia increased significantly, as shown in Figure 4.

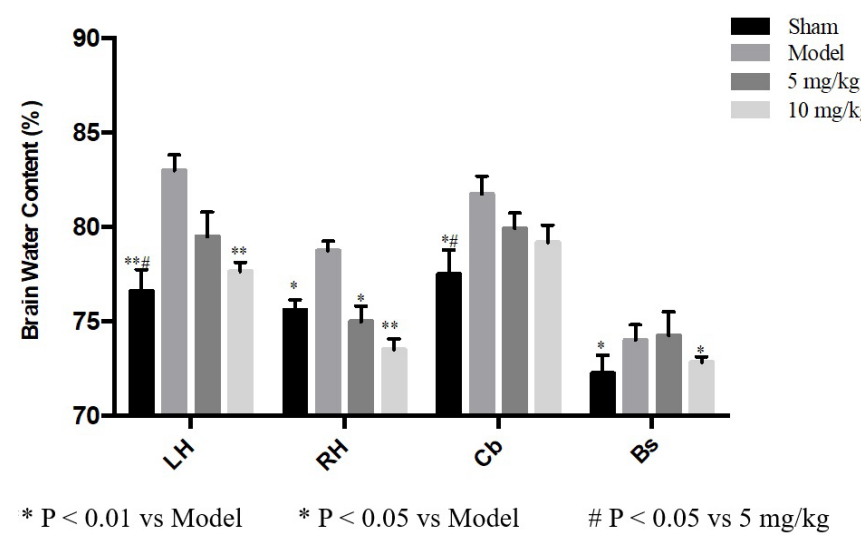

Figure 1. Puerarin alleviated brain edema.

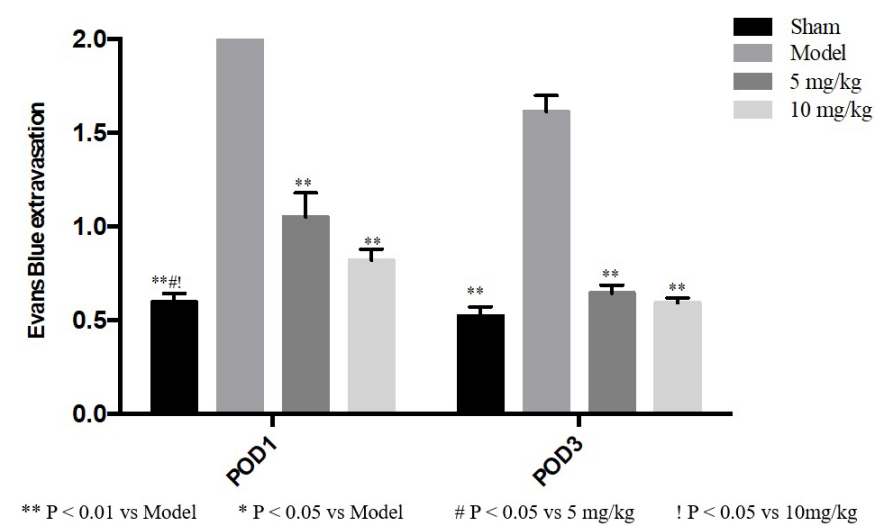

Figure 2. Puerarin protected the blood-brain barrier.

\subsection{The effect of Puerarin on various proteins}

The results showed that Puerarin can inhibit the expressions of Notchl, NICD, RBP-Jא and Hes-1 in SAH of brain tissue, but the content of neuroprotective factor Botch increased, as shown in Figure 5.

\section{Discussion}

A large number of clinical studies and animal research both at home and abroad have confirmed that the inflammatory response plays an important role in the pathological process of early brain injury after SAH. Microglial cells, as the inherent immune phagocytes of the central nervous system, can produce a large number of inflammatory mediators after $\mathrm{SAH}$, thereby activating the nerve inflammation reaction around the damaged tissue. Excessive activation of the inflammation reaction can aggravate the tissue damage. Therefore, some scientists have proposed that by inhibiting the inflammatory factors produced by the activation of microglia after $\mathrm{SAH}$, neuroinflammation can be reduced, thus facilitating a certain protective effect on early brain injury after SAH (Xie et al., 2017; Guo et al., 2016; $\mathrm{Xu}$ et al., 2017).

In our study, the intravenous injection of Puerarin was conducted within 1 hour after SAH. The degree of brain tissue edema, the blood-brain barrier damage, and the number of neuronal cell apoptosis $72 \mathrm{~h}$ after SAH were all detected and evaluated. It was found that early drug treatment after $\mathrm{SAH}$ can improve the neurological dysfunction of rats after $\mathrm{SAH}$, reduce the degree of brain tissue edema and the damage of the blood-brain barrier. Histological staining showed that drug treatment can also reduce the death toll of early neuronal cells.

Since the activation of microglia and neuroinflammation plays an important role in the pathological process of early brain injury after SAH, the experiment also measured the degree of activation of microglia after $\mathrm{SAH}$ through immunofluorescence staining. It is found that, after $\mathrm{SAH}$, microglial cells are obviously
Sham

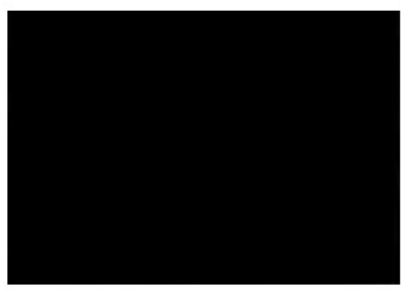

Model
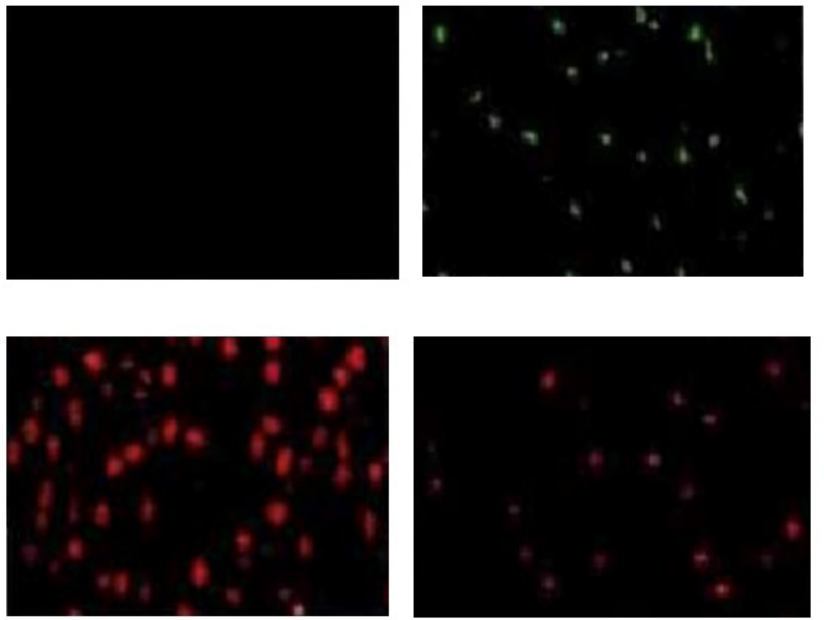

\section{$5 \mathrm{mg} / \mathrm{kg}$}
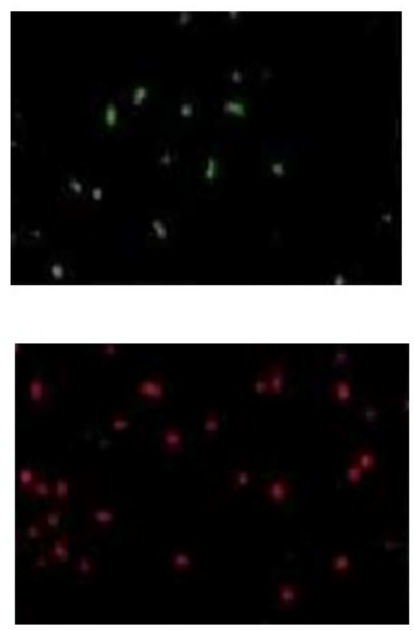

\section{$10 \mathrm{mg} / \mathrm{kg}$}
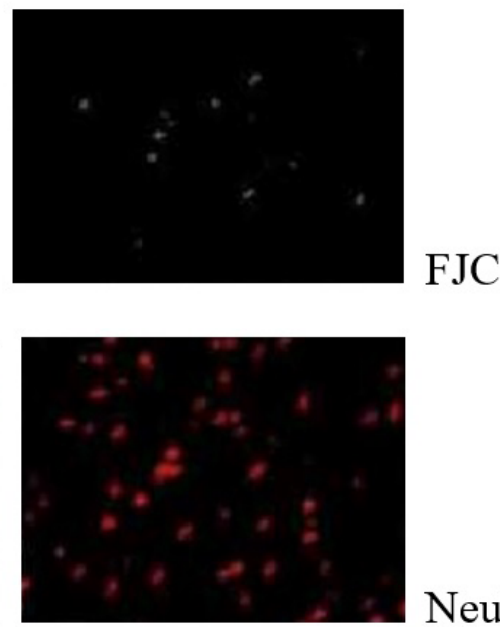

Figure 3. Puerarin protected neuronal cells. 

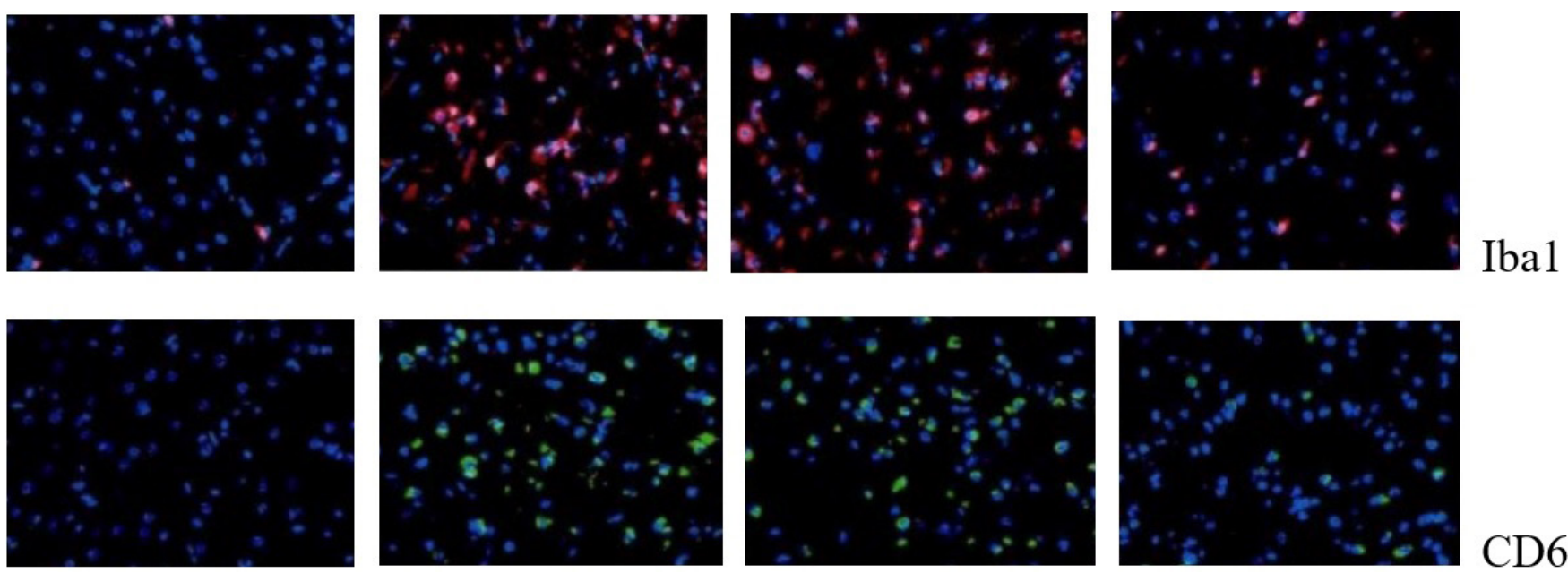

CD68

Figure 4. The effect of Puerarin on the activation of microglia.

\section{Sham \\ Model \\ $5 \mathrm{mg} / \mathrm{kg}$ \\ $10 \mathrm{mg} / \mathrm{kg}$}
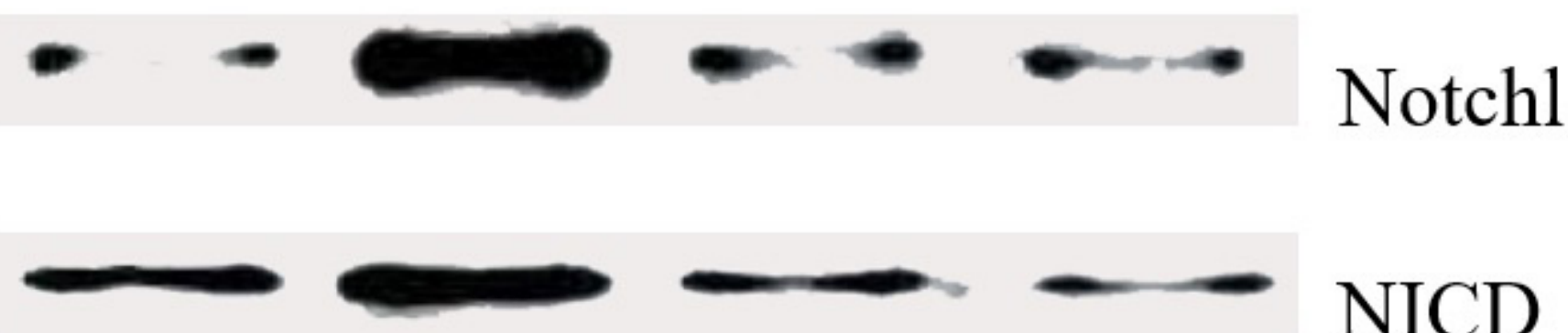

NICD
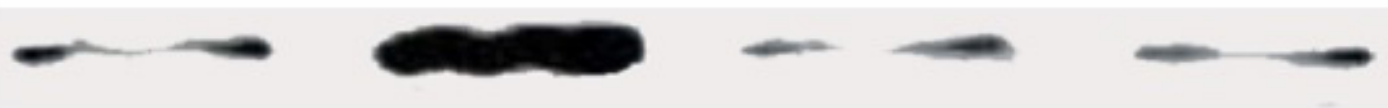

$\mathrm{RBP}-\mathrm{J}_{\kappa}$
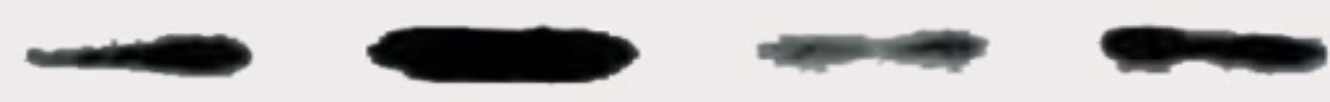

Hes-1
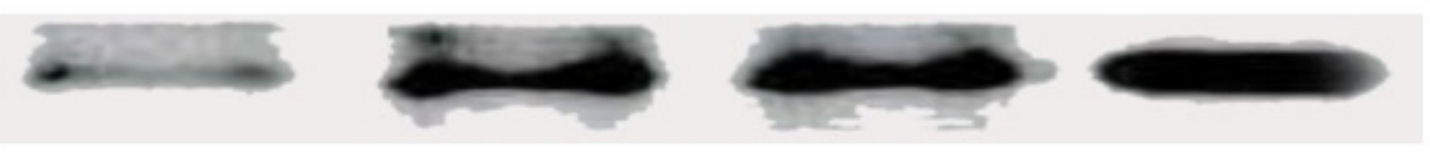

Botch
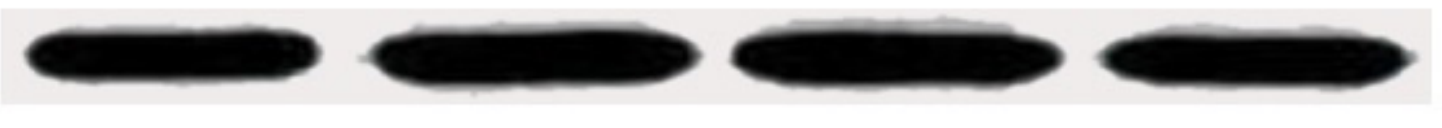

$\beta$-actin

Figure 5. The effects of Puerarin on the expressions of Notchl, NICD, RBP-Jк, Hes-1 and Botch. 
activated, meaning that the proportion of Ibal and CD68 positive microglia cells around the injured brain tissue increased, and the content of inflammatory factors in the damaged brain tissue increased significantly. Early drug treatment can inhibit the activation of microglia. The pathological mechanism after SAH is complicated. Vasospasm and early brain injury are considered as the main complications after SAH. This experiment mainly explored the molecular pathological mechanism of early brain injury after SAH, and opened up new ideas for the treatment of early brain injury after $\mathrm{SAH}$.

Notch is a receptor located on the cell membrane. It was first discovered in Drosophila in vivo studies that the Notch receptor is related to the formation of scratches on the edge of the wings of Drosophila. It was later found existing in multiple species. When its ligands Jaggedl, Jagged2 and Deltal, Delta3, and Delta4 are combined, they can be activated after three shears: the first shearing occurs during the maturation of Notchl. Notchl is cleaved by furin-like invertase in the Golgi, producing two subunits: extracellular regions and transmembrane fragments. These two subunits form Notchl receptors in the form of heterodimers and are localized on the surface of the cell membrane.

The second shearing occurs after Notch binds to the corresponding ligand, and is cleaved into 2 fragments under the action of metalloproteinase/tumor necrosis factor-a converting enzyme. The $\mathrm{N}$-terminal cleavage product is engulfed by the ligand-expressing cells, while the $\mathrm{C}$-terminal cleavage product remains on the membrane and receives the third shearing.

The third shearing product of Notchl was cleaved by a high molecular multiprotein complex, releasing the activated form of Notchl protein NICD (Budohoski et al., 2013; Chi et al., 2012, 2014). After NICD is released from the cell membrane, it enters the nucleus and activates the RBP-Jk and Hes-1 factors in the downstream of the pathway, thereby promoting the expression of cell metabolism-related genes, which in turn regulate cell proliferation, differentiation, synaptic plasticity and neuronal aging. It plays an important role in the process of cell growth and development (Yang et al., 2017a).

Our study employed Western-blot to detect the temporal changes of Notchl and NICD in the early stage after SAH. The results showed that the expressions of Notchl and NICD increased significantly after SAH. Puerarin can reduce the expression of NICD in microglial cells. After the Puerarin treatment, Botch was detected in the brain tissue and found increased after SAH. Drug treatment can further increase the content of Botch in SAH brain tissue.

\section{Conclusion}

In summary, Puerarin can reduce the microglia-mediated neuroinflammatory response after $\mathrm{SAH}$, and the inflammatory response mediated by Notchl receptor activation is involved in the protective effect of Puerarin on brain injury after SAH. By increasing the content of Botch protein in the tissue after $\mathrm{SAH}$, the activation of Notchl receptor is inhibited and EBI is reduced.

\section{Reference}

Ballini, A., Cantore, S., Scacco, S., Coletti, D., \& Tatullo, M. (2018). Mesenchymal stem cells as promoters, enhancers, and playmakers of the translational regenerative medicine. Stem Cells International, 2018, 6927401. http://dx.doi.org/10.1155/2018/6927401. PMid:30510586.

Budohoski, K. P., Czosnyka, M., Kirkpatrick, P. J., Smielewski, P., Steiner, L. A., \& Pickard, J. D. (2013). Clinical relevance of cerebral autoregulation following subarachnoid haemorrhage. Nature Reviews. Neurology, 9(3), 152-163. http://dx.doi.org/10.1038/nrneurol.2013.11. PMid:23419369.

Carbonara, M., Fossi, F., Zoerle, T., Ortolano, F., Moro, F., Pischiutta, F., Zanier, E. R., \& Stocchetti, N. (2018). Neuroprotection in traumatic brain injury: mesenchymal stromal cells can potentially overcome some limitations of previous clinical trials. Frontiers in Neurology, 9, 885. http://dx.doi.org/10.3389/fneur.2018.00885. PMid:30405517.

Chi, Z., Byrne, S. T., Dolinko, A., Harraz, M. M., Kim, M.-S., Umanah, G., Zhong, J., Chen, R., Zhang, J., Xu, J., Chen, L., Pandey, A., Dawson, T. M., \& Dawson, V. L. (2014). Botch is a gamma-glutamyl cyclotransferase that deglycinates and antagonizes Notch. Cell Reports, 7(3), 681-688. http://dx.doi.org/10.1016/j.celrep.2014.03.048. PMid:24767995.

Chi, Z., Zhang, J., Tokunaga, A., Harraz, M. M., Byrne, S. T., Dolinko, A., Xu, J., Blackshaw, S., Gaiano, N., Dawson, T. M., \& Dawson, V. L. (2012). Botch promotes neurogenesis by antagonizing Notch. Developmental Cell, 22(4), 707-720. http://dx.doi.org/10.1016/j. devcel.2012.02.011. PMid:22445366.

Fan, Y., Gao, X., Chen, J., Liu, Y., \& He, J. J. (2016). HIV tat impairs neurogenesis through functioning as a notch ligand and activation of notch signaling pathway. The Journal of Neuroscience : The Official Journal of the Society for Neuroscience, 36(44), 11362-11373. http:// dx.doi.org/10.1523/JNEUROSCI.1208-16.2016. PMid:27807176.

Ferri, A. L. M., Bersano, A., Lisini, D., Boncoraglio, G., Frigerio, S., \& Parati, E. (2016). Mesenchymal stem cells for ischemic stroke: progress and possibilities. Current Medicinal Chemistry, 23(16), 1598-1608. http://dx.doi.org/10.2174/09298673236661602221137 02. PMid:26898654.

Guo, Z., Hu, Q., Xu, L., Guo, Z. N., Ou, Y., He, Y., Yin, C., Sun, X., Tang, J., \& Zhang, J. H. (2016). Lipoxin A4 reduces inflammation through formyl peptide receptor 2/p38 MAPK signaling pathway in subarachnoid hemorrhage rats. Stroke, 47(2), 490-497. http:// dx.doi.org/10.1161/STROKEAHA.115.011223. PMid:26732571.

Minhas, J. S., Panerai, R. B., Ghaly, G., Divall, P., \& Robinson, T. G. (2019). Cerebral autoregulation in hemorrhagic stroke: a systematic review and meta-analysis of transcranial Doppler ultrasonography studies. Journal of Clinical Ultrasound, 47(1), 14-21. http://dx.doi. org/10.1002/jcu.22645. PMid:30270441.

Xie, Y., Guo, H., Wang, L., Xu, L., Zhang, X., Yu, L., Liu, Q., Li, Y., Zhao, N., Zhao, N., Ye, R., \& Liu, X. (2017). Human albumin attenuates excessive innate immunity via inhibition of microglial Mincle/ Syk signaling in subarachnoid hemorrhage. Brain, Behavior, and Immunity, 60, 346-360. http://dx.doi.org/10.1016/j.bbi.2016.11.004. PMid:27845194.

Xu, H., Li, J., Wang, Z., Feng, M., Shen, Y., Cao, S., Li, T., Peng, Y., Fan, L., Chen, J., Gu, C., Yan, F., Wang, L., \& Chen, G. (2017). Methylene blue attenuates neuroinflammation after subarachnoid hemorrhage in rats through the Akt/GSK-3beta/MEF2D signaling pathway. Brain, Behavior, and Immunity, 65, 125-139. http://dx.doi.org/10.1016/j. bbi.2017.04.020. PMid:28457811.

Xu, W., Gao, L., Li, T., Zheng, J., Shao, A., \& Zhang, J. (2018). Apelin-13 alleviates early brain injury after subarachnoid hemorrhage via 
suppression of endoplasmic reticulum stress-mediated apoptosis and blood-brain barrier disruption: possible involvement of ATF6/CHOP pathway. Neuroscience, 388, 284-296. http://dx.doi.org/10.1016/j. neuroscience.2018.07.023. PMid:30036660.

Yang, G., Zhou, X., An, X., Liu, X., Zhang, Y., \& Yu, D. (2017a). Synergistic effect of mild hypothermia and the Notch inhibitor DAPT against post stroke seizures. Biomedicine and Pharmacotherapy, 96, 675-684. http://dx.doi.org/10.1016/j.biopha.2017.10.036. PMid:29035834.

Yang, Y., Ye, Y., Su, X., He, J., Bai, W., \& He, X. (2017b). MSCs-Derived exosomes and neuroinflammation, neurogenesis and therapy of traumatic brain injury. Frontiers in Cellular Neuroscience, 11, 55. http://dx.doi.org/10.3389/fncel.2017.00055. PMid:28293177. 